\title{
Adsorption and onset of lubrication by a double-chained cationic surfactant on silica surfaces
}

\author{
Laurence Serreau, Muriel Beauvais, Caroline Heitz, and Etienne Barthel \\ Laboratoire Surface du Verre et Interfaces, UMR 125, CNRS/Saint-Gobain, 39 \\ quai Lucien Lefranc, B.P. 135, F-93303 Aubervilliers cedex, France
}

\begin{abstract}
In the context of glass fiber manufacturing the onset of lubrication by a $\mathrm{C}_{18}$ doublechained cationic surfactant has been investigated at high normal contact pressures. Comparison with adsorption kinetics demonstrates that lubrication is not directly connected to the surfactant surface excess but originates from the transition to a defect-free bilayer which generates limited dissipation. The impact of ionic strength and shear rate has also been studied.
\end{abstract}

Key words: Friction, lubrication, silica, glass, surfactant, surface modification PACS:

Email address: etienne.barthel@saint-gobain.com (Laurence Serreau, Muriel Beauvais, Caroline Heitz, and Etienne Barthel ). 


\section{$1 \quad 1$ INTRODUCTION}

2 During glass fiber manufacturing, the high friction characteristic of silicate

3 surfaces in water results in surface damage and eventually prejudices the ten-

4 sile strength of the fibers. The necessary lubrication can be imparted through

5 an aqueous dispersion (sizing) which is applied at the initial stage of the glass

6 fiber manufacturing process. The sizing serves many purposes but double-

7 chained cationic surfactants (softeners) are often added to this dispersion to

8 participate in lubrication. However, it is well known that adsorption of sur-

9 factants proceeds slowly [1,2], especially for long-chained amphiphiles [3]. The

10

11

12

question we address in this paper is the kinetics of lubrication: once surfactant adsorption has started, when will lubrication be effective?

It is expected that the answer depends upon the mechanical loading and the friction velocity in a complex manner. In practice, the typical drawing speed is several meters per second, but the contacts between the several hundred glass filaments within one fiber will slide at much slower velocities, which can be in the range of millimeters per second or lower. The filaments slide against each other in the presence of the sizing which initiates both adsorption and lubrication. Such are the operating conditions we emulate in the present study.

Numerous studies have been conducted on the contact and also the friction properties of surfactant covered surfaces. For practical reasons, the bulk of the literature is devoted to short, single chain surfactants, which exhibit faster equilibration [4,516]. Simultaneously, because of their relevance in biological applications, numerous papers deal with the adsorption of lipids. In particular the structure of the surface aggregates and the mechanical response 
of these insoluble double-chained surfactants have been studied in great details [6,7,8,8,10].

In this paper, we investigate the early stages of lubrication just after immersion of silica surfaces in an aqueous dispersion of a typical double-chained $\left(2 \mathrm{C}_{18}\right)$ cationic surfactant. The surfactant dispersion was investigated by Small Angle Xray Scattering (SAXS) and Static Light Scattering (SLS) and the adsorption kinetics on silica surfaces by Attenuated Total Reflection (ATR) Fourier Transform Infrared (FTIR) spectroscopy. Using atomic force microscopy (AFM) and macroscopic friction tests, we have measured the contact properties (repulsive barrier, adhesion and friction) of macroscopic silica surfaces in the initial stages of adsorption as a function of time after immersion. The results highlight the impact of the adsorption kinetics and the changes of the surfactant configuration during the early stages of adsorption. The picture which emerges is that of a gradual transition from a disordered adsorbed layer with high friction to a lubricating defect-free bilayer. Shear is shown to play a role in the transition to the lubricating state.

\section{EXPERIMENTAL SECTION}

\subsection{Materials}

The double-chained cationic surfactant 1-methyl-2-noroleyl-3-oleic acid-aminoethylimidazolinium methosulfate (DOAIM, Figure 1) in isopropanol (25\% wt) is obtained from Goldschmidt Rewo GmbH \& Co.,(Germany) and used as received. The molecular weight is $740 \mathrm{~g} / \mathrm{mol}$ and the density $0.97 \mathrm{~g} / \mathrm{cm}^{3}$. The CMC with isopropanol measured by surface tension is $1 \times 10^{-5} \mathrm{M}$. The chain 
melting temperature is $46^{\circ} \mathrm{C}$ as measured by DSC, in agreement with the values obtained for similar compounds [11]. All the experiments were performed at ambient temperature.

Solutions of DOAIM at $5 \times 10^{-4} \mathrm{M}$ or $1 \times 10^{-3} \mathrm{M}$ were prepared in milli $\mathrm{Q}$ water with 24 hours gentle stirring after evaporation of the isopropanol at 60C. Most experiments were carried out at natural $\mathrm{pH} \simeq 4.6$. In a set of experiments, the ionic strength was varied with acetic acid/sodium acetate while maintaining constant $\mathrm{pH}=4.5$. Such concentration and $\mathrm{pH}$ conditions are typical for actual sizing formulations.

\subsection{Methods}

\subsubsection{Equilibrium characterization}

The SAXS experiments were performed in a Kratky set-up (Anton Paar) with a $\mathrm{Cu} \mathrm{K}_{\alpha}$ source $(0.1542 \mathrm{~nm})$ and a linear gaz detector placed at $23 \mathrm{~cm}$ from the source. The SLS experiments were performed on a Malvern Zetasizer 3 equipped with an He-Ne laser (633 nm), a photomultiplier and a goniometer. The same piece of equipment was used to measure the zeta potential by electrophoretic mobility in a liquid cell. The laser interferometric comb method was used. The test system was $200 \mathrm{~nm}$ diameter silica particles (Stœeber synthesis). Adsorption at a given concentration was carried out by dilution from a $10^{-4} \mathrm{M}$ surfactant solution followed by 5 hour equilibration time. Surface tension was measured by the Wilhelmy plate method. 
${ }_{92} \quad A=k \epsilon\left[\frac{c_{s} d_{p}}{2}+\Gamma\right]$

\subsubsection{FTIR/ATR adsorption kinetics}

Adsorption kinetics were measured by FTIR spectroscopy in the ATR mode using a Nicolet Nexus 670 spectrometer equipped with a MIR source, a $\mathrm{KBr}$ beamsplitter and a MCT-A detector. The experiments were carried out on a germaniun internal reflection element (trapezoidal, $50 \times 10 \times 1 \mathrm{~mm}^{3}, 45^{\circ}$ incident angle) covered on the larger side by a silica layer $\simeq 7 \mathrm{~nm}$ thick deposited by magnetron sputtering. Before use, the surfaces were cleaned with a sequence of detergent solution, deionized water, acetone and absolute ethanol for 15 minutes in an ultrasonic bath, followed by a final UV/Ozone treatment for $1 \mathrm{~h}$. After cleaning, the wafer was introduced in the internal multi-reflection cell which was immediately assembled and aligned in the sample compartment of the spectrometer. A peristaltic pump and a three-way valve were used to circulate either the pure solvent or the surfactant solution through the flow cell. Spectra were taken at a resolution of $4 \mathrm{~cm}^{-1}$ for 8,32 or 128 scans. A background spectrum was collected after the cell was filled with water, before the surfactant solution was pumped in. Following Harrick [12]13]14]15]16], the amount of adsorbed surfactant can be quantified from the absorbance of some vibration band of the molecule. In our case we have followed the evolution of the $\mathrm{CH}_{2}$ bands between 2800 and $3000 \mathrm{~cm}^{-1}$. Absorbance of the vibration band $\nu_{s}\left(\mathrm{CH}_{2}\right)$ at $2854 \mathrm{~cm}^{-1}$ is used to determine the surface excess as a function of time. This band has been chosen because it is less affected by the baseline drift associated with the strong band of water in the range 3200-3300 $\mathrm{cm}^{-1}$. The surface excess $\Gamma$ is calculated from [12]14] 
93

${ }_{94} \quad k=\frac{n_{2} E_{0}{ }^{2}}{n_{1} \cos \theta} N$

where $d_{p}$ is the penetration length of the evanescent wave, $\lambda$ the wavelength, $N$ the number of internal reflections, $E_{0}$ the electric field amplitude, $n_{1}$ and $n_{2}$ the refractive index of the germanium and the solution respectively, $\theta$ the incident angle, $A$ and $\epsilon$ respectively the absorbance and molecular extinction coefficient of the vibration band considered, and $c_{s}$ the concentration of the absorbing species in solution. Assumption is made that $c_{s}$ is not modified by adsorption. In practice $N$ and $E_{0}$ cannot easily be determined so that $k$ is determined from relation (11) by a calibration with a non adsorbing compound of known extinction coefficient (tert-butanol).

\subsubsection{AFM Surface forces measurement}

AFMs have been used for surface forces measurements in various environments [17,18,19]. Here the experiments were performed on a Nanoscope III (Digital Instrument) with a silicon nitride tip using a liquid cell. Prior to the experiment, the tip was cleaned by irradiation for 60 minutes in a UV-ozone flow. A typical AFM experiment starts with a control of the tip shape quality and the silica surface cleanliness by measuring interaction forces between the AFM tip and the silica surface in milli-Q water. The DOAIM solution is then introduced and the surface forces profiles between tip and silica substrate are recorded every 3 minutes. 


\subsubsection{Friction experiments}

Friction experiments have been performed on two reciprocating ball-on-plate tribometers: for low pressure friction measurements, a home built millitribometer with a $50 \mathrm{mN}$ load range and a $0.02 \mathrm{mms}^{-1}$ maximum sliding velocity was used; for a larger friction velocity range, a commercial (Plint T79) tribometer with sliding velocity ranging from 0.01 to $10 \mathrm{mms}^{-1}$. However, for this latter equipment, the normal load ranges between 0.1 and $20 \mathrm{~N}$ which results in larger mean pressures. The plate is a silica, $2 \mathrm{~mm}$ thick substrate optically polished on both sides (GE quartz). The ball is a fused silica sphere made from silica rods (GE quartz). The end of the rod was melted with a blowtorch until a molten droplet of glass formed with a radius of 2 to $4 \mathrm{~mm}$. Both surfaces were cleaned before use with a detergent-water-absolute ethanol sequence for 15 minutes in an ultrasonic bath.

To emulate lubrication in the presence of the sizing, the friction experiments were all conducted in the presence of the aqueous surfactant dispersion, inside a liquid cell. It is also important to note that to minimize and control the impact of shear, the typical friction experiments were not conducted as continuous runs as is usually done for such measurements: on the contrary, unless otherwise stated, the surfaces were brought into contact every 5 minutes for a series of two cycles only, typically lasting a few seconds and were then separated again (Fig. (2). The friction force was measured by averaging on the second cycle. When separated, care was taken that the silica surfaces remained immersed in the solution until the next measurement. For each experiment, the friction coefficient was first measured between surfaces immersed in pure water. The water was then removed and replaced by the solution under study. The first point in each friction graph is therefore the friction coefficient in pure 
water.

\section{Results}

\subsection{Characterization of the solution and adsorption}

The pure surfactant system (after extraction of the isopropanol) is optically birefringent. The SAXS diffractogram exhibits one single, fine Bragg peak (Fig. 3) typical for an $\mathrm{L}_{\beta}$ phase. The repeat distance is $3.31 \mathrm{~nm}$. After dilution in water $(1 \mathrm{M})$, the system exhibits shear induced birefringence which persists over days. In the SAXS diffractogram, a series of equally spaced peaks is recorded (Fig. 3). These features are also typical for a lamellar phase. The first order diffraction peak has moved to smaller wave vector and the repeat distance has increased to $7.85 \mathrm{~nm}$, which is fully consistent with the $7.65 \mathrm{~nm}$ value expected for dilution of the lamellar phase to $1 \mathrm{M}$. Upon further dilution the Xray signal and the optical birefringence is lost. Around $1 \times 10^{-3} \mathrm{M}$, well above the CMC, the bilayer conformation is also evidenced optically by the presence of multilamellar vesicles. At lower concentrations, SLS experiments were carried out. The scattered intensity recorded for $2.5 \times 10^{-3}$ and $1.0 \times 10^{-4} \mathrm{M}$ are displayed on Fig. 4. Beyond the quadratic behaviour for small diffusion wave vectors, the static correlation function exhibits a moderate decay. The full shape of the correlation function is consistent with extended disks [20] as expected for large dilutions where the correlation between lamel-

lae is lost. The measured correlation length are 310 and $550 \mathrm{~nm}$ for $2.5 \times 10^{-3}$ 
and $1.0 \times 10^{-4} \mathrm{M}$, showing that the materials behaves as sheets at lengthscales smaller than the correlation length. In conclusion, the surfactant solution exhibits a lamellar phase resulting from the bilayer association of the individual surfactant molecules and the bilayer structure is preserved upon dilution.

From the surface tension as a function of concentration, we determined a critical micelle concentration $\mathrm{CMC}=9 \times 10^{-6} \mathrm{M}$ and an area per head $A_{H}=0.71 \mathrm{~nm}^{2}$. The results of the zeta potential measurements at natural $\mathrm{pH}$ are displayed on Fig. [5. The zeta potential of the bare silica spheres is found at the expected $-60 \mathrm{mV}$ value. Upon adsorption, the surface charge decreases and is finally reversed at the point of zero charge $\mathrm{PZC}=5 \times 10^{-6} \mathrm{M}$ well below the $\mathrm{CMC}$. This charge reversal behavior is characteristic for the adsorption of a bilayer at the surface.

\subsection{Friction - Time effect}

A first series of friction experiments were carried out at low contact pressures (concentration $\mathrm{C}=10^{-3} \mathrm{M}$, natural $\mathrm{pH}$, sliding velocity $\mathrm{v}=0.014 \mathrm{mms}^{-1}$, mean contact pressure $\mathrm{P}_{m}=90 \mathrm{MPa}$, Figure $[$ ). The typical friction coefficient of silica surfaces immersed in pure water is $0.6 \pm 0.1$ with a variability due to surface preparation. Typical friction coefficients after five minutes of immersion in the DOAIM solution $\left(\mathrm{C}=10^{-3} \mathrm{M}\right)$ are down to 0.50 which indicates negligible (though measurable) lubrication. On the other hand, if the surfaces are first immersed 15 hours in a solution of DOAIM $\left(10^{-3} \mathrm{M}\right)$ before the friction experiment starts (procedure C) then the measured friction coefficient is lower than 0.1, and sometimes reaches 0.03 , revealing fully lubricated surfaces. Similar results are recorded for $\mathrm{C}=5 \times 10^{-4} \mathrm{M}$. The long equilibration 
time in procedure $\mathrm{C}$ is typical for surfactant lubrication experiments [21,22].

A friction coefficient of about 0.1 or lower is usual for surfactant lubrication, especially for double-chained surfactants with long chains [8,23],24]. However, when the same friction experiment starts immediately after immersion in the surfactant solution (procedure A), the friction coefficient decreases to reach the low friction value (about 0.05) after only ca 2 hours. The transition is not linear with time. With repeated friction tests carried out every $5 \mathrm{~min}$ we observe an initial plateau at the high friction value around 0.5-0.6, which typically lasts 1 hour before the friction coefficient starts to decrease by one order of magnitude down to values around 0.05 . These results exemplify the fact that lubrication with double-chained surfactants is not instantaneous but starts after an induction period.

\subsection{AFM surface forces measurements}

In the AFM force measurements, the force vs distance curve (Figure 7 , inset) first displays a repulsive long range interaction, followed by a steeper repulsive interaction starting around $10 \mathrm{~nm}$. The former has not been quantified due to the low signal to noise ratio but the results are consistent with the electrostatic double layer interaction demonstrated by the zeta potential measurements (Fig. [5). The latter is due to the mechanical compression of the bilayer. Then, for distances close to 3-4 nm, the AFM tip jumps into contact. This jumpin distance of 3-4 $\mathrm{nm}$ is close to the thickness of a DOAIM bilayer. Such a behaviour is well-known in the literature [4,25,26,27]. The jump-in force, defined as the repulsive force at jump-in, is an estimate of the mechanical resistance of the bilayers. Pulling the tip back induces the rupture of the 
tip-surface contact: a negative force is registered which signals adhesion (not shown on the inset). The amplitude of this pull-out force is a measure of the tip-surface adhesion energy [28,29]. The jump-in force in a solution of DOAIM $\left(1 \times 10^{-3} \mathrm{M}\right)$ has been measured as a function of time, as well as the pull-out force (Figure 7). We note that the jump-in and the pull-out forces measured here are tightly correlated, as already reported in the literature [21]. The jump-in force (counted positive) increases as the magnitude of the pull-out force (counted negative) decreases. They obey a time evolution similar to the friction coefficient: in procedure A, it stays constant for about one hour before the decrease to low friction; similarly jump-in and pull-out forces exhibit an initial plateau before a transition around $60 \mathrm{~min}$ to equilibrium values with large jump-in force and negligible adhesion. More complete results on the adsorption isotherm measured by ellipsometry and the mechanical response of the surfactant bilayer at equilibrium obtained with a Surface Forces Apparatus will be published separately.

\subsection{Adsorption kinetics of DOAIM}

In this context, it is interesting to correlate the time evolution of the contact properties with the amount of surfactant adsorbed on the surface (surface excess). A measurement of the adsorption kinetics $\left(5 \times 10^{-4} \mathrm{M}\right.$, FTIR-ATR $)$ is shown on Figure 8. Two different regimes are observed and the data is reasonably well fitted by a double exponential function with a fast time constant $\tau_{1}=25 \mathrm{~min}$ and a slow time constant $\tau_{2}=205 \mathrm{~min}$, leading to a pseudo-plateau. At pseudo-saturation, the adsorbed amount is $6.2 \mu \mathrm{molm}^{-2}$. Rinsing with recirculating water leads to little desorption, down to $5.5 \mu \mathrm{molm}^{-2}$. The area 
per molecule determined from surface tension measurements is $0.79 \mathrm{~nm}^{2}$. From this value we conclude that at saturation, a full bilayer is formed at the silica surface. A comparison of Figures 6 and 7 with Figure 8 demonstrates that during the induction period, when the friction coefficient is high and constant, the adsorption of the surfactant is fast. When the transition to the lubricated state occurs (procedure A), we can estimate that the surface excess is already roughly as large as half a bilayer. From this observation we conclude that there is no simple proportionality relation between the adsorbed amount and the friction coefficient in this regime but that a more complex mechanism is called for to explain the onset of lubrication. In order to gain a clearer view of this mechanism, we have performed a series of experiments to probe the impact of kinetic parameters on the lubrication of the surfaces.

\subsection{Impact of shear on the onset of lubrication}

We measured the friction when the experiment starts only three hours after immersion in the solution (procedure B, Figure 6). In such conditions, the adsorption is almost complete (the surface excess amounts to $85 \%$ of the maximum, Figure 8) and a lubricated surface is obtained following procedure A. If lubrication were only controlled by the adsorption of the surfactant, then a low friction coefficient would be expected, as with procedure C. In contrast, a trend similar to procedure A is observed: after 3 hours of induction, we measure an initial value of the friction coefficient of approximately 0.5 . Transition towards a low friction coefficient is observed around 1.5 hours after the friction experiment has started, and a low value (0.07) is reached about 2 hours after the beginning of the friction experiment, that is a total of about 5 hours after 
immersion. This result attests to the fact that shear accelerates the onset of lubrication: low friction is obtained after 2 hours in procedure A, in which friction is probed every 5 minutes, but only after 5 hours in procedure B, where the system is completely at rest for the first 3 hours.

\subsection{Ionic strength effect}

Ionic strength also impacts the adsorption process. In the presence of salt $(\mathrm{pH}$ 4.5), the adsorption kinetics is much faster (Figure 8, inset) and for an ionic strength of $2 \times 10^{-2} \mathrm{M}$, half-coverage of the surface is reached within minutes. Figure 9 shows the evolution of the friction coefficient with time (DOAIM $\left.5 \times 10^{-4} \mathrm{M}, \mathrm{P}_{m}=320-340 \mathrm{MPa}, \mathrm{pH}=4.5\right)$ for different salt concentrations. The experiments follow procedure A where friction starts immediately after immersion. We observe that the transition towards low friction is considerably faster, a trend similar to the adsorption kinetics. The initial friction plateau has now been suppressed and the time to reach the lubricating state decreases when the ionic strength increases. For the highest salt concentration, the initial value of the friction coefficient after 5 minutes of immersion is already 3 times lower than in pure water. Note that the friction spike to 0.2 which follows the first low friction data point (Figure 9) is a reproducible feature presumably connected to more dissipative intermediate configurations towards a fully lubricated surface. Similarly at high ionic strength the AFM force curves demonstrate an almost instantaneous build-up of the repulsive force wall (not shown). 


\subsection{Sliding velocity}

We have demonstrated in section 3.5 that shear accelerates the transition to the lubricated state. A final set of experiments aims at exploring the impact of shear rate. Friction experiments at different sliding velocities were performed $\left(\mathrm{P}_{m}=320-340 \mathrm{MPa}\right)$ following procedure A. For the lower velocity $\left(0.01 \mathrm{mms}^{-1}\right)$, the global evolution is similar to the evolution recorded at lower mean pressure in contact $(90 \mathrm{MPa})$ : the high (0.53) initial friction coefficient decreases with time to reach a stable value of 0.06 . However, we can perform experiments in a wider velocity range only at higher loads (section 2.2.4). This is why the transition towards low friction coefficient is achieved after 5 hours instead of 2. For high velocities, the equilibrium configuration is reached much faster, within 5-10 minutes. Figure 10 summarizes the main effects of the sliding velocity on the initial and final values of the friction coefficient. Note that the magnitude of the lubrication effect also decreases, since the value of the friction coefficient of silica surfaces in pure water decreases with the sliding velocity [30].

\section{Discussion}

\subsection{The generic lubricating state}

In the lubricated state, the friction coefficient is as low as 0.05 , as observed for instance in procedure $\mathrm{C}$, after a 15 hour adsorption period. For the types of loading used here, the mean normal pressures in the contact are significantly larger than typical hemifusion thresholds for double-chained $\mathrm{C}_{18}$ surfactants 
(several $10 \mathrm{MPa}$ ) [31]. As a result, hemifusion of the bilayers present on both surfaces occurs and this low value for the friction coefficient results from the friction between two hydrocarbon monolayers (Figure11]b'). In this lubricated state, the average interfacial shear stress $\tau$ is of the order of $20 \mathrm{MPa}$ and is little affected by the sliding velocity. Such a value is typical for monolayer-monolayer contacts in air 24] and is consistent with an approximate model connecting friction and adhesion hysteresis $\Delta \gamma$ [23]. Indeed it has been proposed that

$$
\tau \simeq \Delta \gamma / \delta
$$

where $\delta$ is a molecular dimension. Reasonable values are $\Delta \gamma \simeq 10 \mathrm{mJm}^{-2}$ and $\delta \simeq 1 \mathrm{~nm}[24]$, so that $\tau \simeq 10 \mathrm{MPa}$. Friction between the outermost surfaces of the two pristine bilayers would lead to much lower friction coefficients: values one order of magnitude lower, as low as 0.004, were reported for instance for gemini surfactants [22].

\subsection{Organisation during adsorption}

As amply demonstrated by our present results, this configuration is not readily obtained upon adsorption from the solution. Indeed, the equilibrium configuration in the bulk is usually different from the equilibrium configuration of the surfactant aggregates adsorbed on a surface and the same surface excess may lead to very different surfactant conformations, with either dissipation and friction or lubrication. Subtle effects control the surfactant conformation after adsorption [32,33, 34, 35, 36].

This is especially true when the interaction is strong, which is the case when surface and surfactant are oppositely charged: for cationic surfactants, the 
electrostatic interaction with the negatively charged silica surface results in fast initial adsorption (Fig. 8). After this first adsorption stage, reorganization is required. For example, Chattoraj and Biswas [2] observed two characteristics times for the adsorption kinetics of short-chained cationic surfactants on silica surfaces. The mechanism they propose is as follows: in the first step, surfactant molecules from the bulk diffuse to the surface and adsorb quickly with random orientation onto the silica surface; in the second step, the crowded molecules tend to re-orient in a regular fashion leading to the formation of adsorbed patches of surface micellar aggregates. Such configuration changes will create more vacant spaces for further adsorption of surfactant from the bulk to the surface. Similarly, for adsorption of CTAB above the cmc on mica surfaces, Chen et al. [1] propose a slightly different model where micelles adsorb directly on the surfaces and subsequently reorganize. The idea is supported by the fact that the same density of molecules is measured in the adsorbed layer and in the micelles in solution. DOAIM, as many double-chained $\mathrm{C}_{18}$ surfactant, is dispersed as bilayers as further demonstrated by the present optical, SAXS and SLS results. An adsorption process similar to lipid vesicle deposition must therefore be considered: the charged vesicles present in the solution will adsorb quickly as patches of bilayers and in an uncorrelated way [38,39. Rearrangement must proceed before a defect-free bilayer is obtained [15]. This scenario parallels the mechanism proposed by Chen [1] but here the rearrangement is expected to be slower: the characteristic times for adsorption are considerably larger than for short chain surfactants [2,4] since for long chain surfactants, below the chain melting temperature, reorganization is hampered by the slow dynamics [3]. 


\subsection{Bilayers, depletion and contact properties}

The defective nature of the surfactant layer in the initial stage of adsorption strongly impacts its mechanical response. SFA experiments have shown that lipid bilayers exhibit a smaller jump-in force and a larger pull-out force when depleted, i.e. depletion facilitates hemifusion and increases adhesion [21]. Along the same line of thought, hemifusion in the SFA has been shown to correlate with defect density (monolayer or bilayer holes) as identified by AFM [40]. The defect density was controlled by the deposition pressure in the LangmuirBlodgett trough. Similar studies have been reported for the mechanical response of lipid bilayers measured by AFM as a function of surface excess. AFM experiments have shown that depletion [8] and ionic strength [6] impact friction. The results were somehow discussed in terms of packing density. For lipid bilayers, an interesting suggestion is that the reduced stability results from the increased hydrophobic interactions between depleted bilayers [21, not from a simple decrease in the density. Similarly, for shorter single-chained surfactant it has also been observed either by SFA or AFM force measurements that near the CMC, when the surfaces are pushed to bilayer contact, the jump-in force increases with surfactant concentration while the adhesion is maximum for monolayer coverage [16,41]. A connexion between micellization energy and mechanical resistance at equilibrium has also been established [27].

These observations all converge to demonstrate that an increase in the packing density of molecules in the outer layer of the bilayer leads to enhanced stability and reduction of adhesion. In the present experiments the results demonstrate an increase of the jump-in force and a reduction of adhesion as a function of time (Fig. 7). For DOAIM adsorbed at concentrations significantly larger than 
the $\mathrm{CMC}$, the initial conformation is characteristic of frustrated aggregates adsorbed at the surface (Fig. 11, a), which we loosely call defective bilayer. Our results are in complete agreement with the picture of a gradual healing of the initially defective bilayer.

\subsection{Friction and onset of lubrication}

Initially, before the defect-free bilayer is formed, a large friction coefficient is recorded, around 0.5. Compared to the bare silica-silica friction coefficient, this value demonstrate a very moderate impact of the adsorbed surfactant. We suggest that this sizeable interfacial shear results from the dissipation which accompanies the deformation of the aggregates present at the surface. These deformations may be transitions from bilayer to tilted bilayer, aggregate ruptures, etc (Fig. 11, a'). Defective bilayers give rise not only to easier hemifusion and enhanced adhesion, but also to friction because they allow for more deformation at the molecular scale. The results are similar to the large friction recorded for lipid bilayers in AFM experiments when a second mechanical transition threshold is reached, well above hemifusion, and for which "direct surface contact" is evoked [6,8,38].

The decrease towards low friction is typically observed after 1 hour (Fig [6). In parallel the surface forces exhibit a decrease in the adhesion force and the repulsive jump-in force becomes more pronounced (Fig. [7). This trend we connect with the organization at the surface which evolves to a structure closer to a more ordered, stable, bilayer exposing fewer hydrophobic moieties. The transition at the local scale from a defective towards a stable bilayer has been completed. Indeed the friction (Fig. 6) and adhesion (Fig. 7) drops recorded 
here are consistent with Eq. 4.

There remains to be explained why the transition is abrupt and does not directly correlate with the surface excess. It is possible that a critical flaw size exists below which the pressure-induced transition to a tilted or disorganized layer is prevented. This concept parallels the theory for bilayer stability [42,43]. If the flaw density is large enough, as occurs initially, the full surface induces dissipation through aggregate edges. It is only when sufficient healing has occurred and some defect-free patches have formed that the overall friction coefficient decreases. In this scheme, the lubricated state results from stabilisation of the surfactant layer through healing of the larger defects.

The transition towards the lubricated state occurs faster in the presence of salt, because ionic strength screens long range electrostatic double layer interactions and facilitates rearrangement. Similarly, we have observed that the transition towards lubricating state occurs earlier in time when the system is submitted to friction immediately after immersion. Higher sliding velocity also accelerates the transition. We conclude that shear and/or contact due to the friction experiment itself favors the bilayer organization of the surfactant between the two surfaces [41]. Indeed shear provides the symetry breaking driving force which promotes layering [44,45] as well as the mechanical energy which activates structural transitions [46]. It favours surfactant accumulation and lamellar ordering turning the adsorbed material into a fully formed bilayer 9,41. 
421

422

423

\section{Conclusion}

The friction coefficient between millimetric silica surfaces was measured during adsorption of a $\mathrm{C}_{18}$ double-chained surfactant. A transition from high to low friction is observed which parallels the contact properties measured with the AFM. The results are not directly correlated to the surface excess. They point to the role of the organisation of the surfactant into a defect-free bilayer for lubrication to be effective. Lubrication is obtained faster at higher ionic strength and under shear because both facilitate the bilayer organization. We have also study the impact of addition of other surface active sizing components such as silanes on surfactant lubrication. Strong effects have been evidenced due to interaction and/or competitive adsorption, which have been published separately [47].

\section{Acknowledgements}

We thank M. Clerc-Imperor and R. Roquigny for the SAXS experiments.

\section{References}

[1] Y.L. Chen, S. Chen, C. Frank, and J. Israelachvili. J.Colloid Interf. Sci., 153 (1992) 244.

[2] S.C. Biswas and D.K. Chattoraj. J. Colloid Interf. Sci., 205 (1998) 12.

[3] W. A. Hayes and D. K. Schwartz. Langmuir, 14 (1998) 5913.

[4] V. Subramanian and W. Ducker. J. Phys. Chem. B, 105 (2001) 1389. 
[5] I. U. Vakarelski, S. C. Brown, Y. I. Rabinovich, and B. M. Mougdil. Langmuir, $20(2004) 1724$.

[6] G. Oncins, S. Garcia-Manyes, and F. Sanz. Langmuir, 21 (2005) 7373.

[7] R. M. Pashley, P. M. McGuiggan, B. W. Ninham, J. Brady, and D. F. Evans. J. Phys. Chem., 90 (1986) 1637.

[8] L. M. Grant and F. Tiberg. Biophys. J., 82 (2002) 1373.

[9] K. Boschkova, A. Feiler, B. Kronberg, and J. J. Stalgren. Langmuir, 18 (2002) 7930.

[10] K. Boschkova, B. Kronberg, J. J. Stalgren, K. Persson, and M. Ratoi-Salagean. Langmuir, 18 (2002) 1680.

[11] Y. Liu and D. F. Evans. Langmuir, 12 (1996) 1235.

[12] N.J. Harrick. J. Phys. Chem., 64 (1960) 1110.

[13] R. P. Sperline, S. Muralidharan, and H. Freiser. Langmuir, 3 (1987) 198.

[14] M.-J. Azzopardi and H. Arribart. J. Adhes., 46 (1994) 103.

[15] D. J. Neivandt, M. L. Gee, M. L. Hair, and C. P. Tripp. J. Phys. Chem. B, 102 (1998) 5107.

[16] P. K. Singh, J. J. Adler, Y. I. Rabinovich, and B. M. Moudgil. Langmuir-, 17 (2001) 468 .

[17] W. A. Ducker, T. J. Senden, and R. M. Pashley. Langmuir, 8 (1992) 1831.

[18] S. Sounilhac, E. Barthel, and F. Creuzet. Appl. surf. sci., 140 (1999) 411.

[19] S. Sounilhac, E. Barthel, and F. Creuzet. J. Appl. Phys., 85 (1999) 222.

[20] B. Zhmud and F. Tiberg. Adv. Coll. Interface Sci., 113 (2005) 21. 
[21] C. A. Helm, J. N. Israelachvili, and P. M. McGuiggan. Biochem., 31 (1992) 1794.

[22] C. Drummond, J. Israelachvili, and P. Richetti. Phys. Review E, 67 (2003) 066110-1-16.

[23] S; Yamada and J. Israelachvili. J. Phys. Chem. B, 102 (1998) 234.

[24] W. H. Briscoe, S. Titmuss, F. Tiberg, R. K. Thomas, D. J. McGillivray, and J. Klein. Nature, 444 (2006) 191.

[25] J. J. Adler, P. K. Singh, A. Patist, Y. I. Rabinovich, D. O. Shah, and B. M. Moudgil. Langmuir, 16 (2000) 7255.

[26] Y. I. Rabinovich, I. U. Vakarelski, S. C. Brown, P. K. Singh, and B. M. Moudgil. J. Colloid Interface Sci., 270 (2004) 29.

[27] Y. I. Rabinovich, S. Pandey, D. O. Shah, and B. M. Moudgil. Langmuir, 22 (2006) 6858.

[28] J. N. Israelachvili. Intermolecular and Surface Forces. Academic Press, San Diego, 1992.

[29] E. Barthel. J. Phys. D: Appl. Phys., 41 (2008) 163001.

[30] G. Di Toro, D. L. Goldsby, and T. E. Tullis. Nature, 427 (2004) 436.

[31] C. A. Helm, J. N. Israelachvili, and P. M. McGuiggan. Science, 246 (1989) 919.

[32] S. Manne and H. E. Gaub. Science, 270 (1995) 1480.

[33] E. J. Wanless and W. A. Ducker. J. Phys. Chem., 100 (1996) 3207.

[34] R. E. Lamont and W. A. Ducker. J. Am. Chem. Soc., 120 (1998) 7602.

[35] R. Atkin, V.S.J. Craig, E.J. Wanless, and S. Biggs. Adv. Colloid Interf. Sci., $103(2003) 219$. 
[36] S. Paria and K. C. Khilar. Adv. Colloid Interf. Sci., 110 (2004) 75.

[37] R. P. Richter and A. Brisson. Langmuir, 19 (2003) 1632.

[38] R. P. Richter and A. Brisson. Langmuir, 19 (2003) 1632.

[39] R. P. Richter, R. Berat, and A. R. Brisson. Langmuir, 22 (2006) 3497.

[40] M. Benz, T. Gutsmann, N. Chen, R. Tadmor, and J. Israelachvili. Biophys. J., $86(2004) 870$.

[41] R. W. Rutland and J. L. Parker. Langmuir, 10 (1994) 1110.

[42] B. N. J. Persson and E. Tosatti. Phys. Rev. B, 50 (1994) 5590.

[43] E. Barthel. Thin Solid Films, 330 (1998) 27.

[44] J. Zipfel, J. Berghausen, G. Schmidt, P. Lindner, P. Alexandridis, M. Tsianou, and W. Richtering. Phys. Chem. Chem. Phys., 1 (1999) 3905.

[45] Y. Li, Y. Golan, A. Martin-Herranz, O. Pelletier, M. Yasa, J. N. Israelachvili, and C. R. Safinya. Int. J. Thermophys., 22 (2001) 1175.

[46] M. Akbulut, C. Nianhuan, N. Maeda, J. Israelachvili, T. Grunewald, and C. Helm. J. Phys. Chem. B, 109 (2005) 12509.

[47] M. Beauvais, L. Serreau, C. Heitz and E. Barthel. J. Colloid Interf. Sci. in press, doi:10.1016/j.jcis.2008.11.036. 


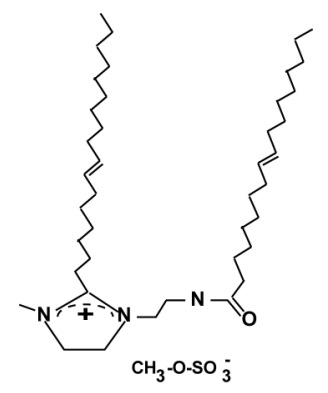

Fig. 1. Chemical structure of the DOAIM

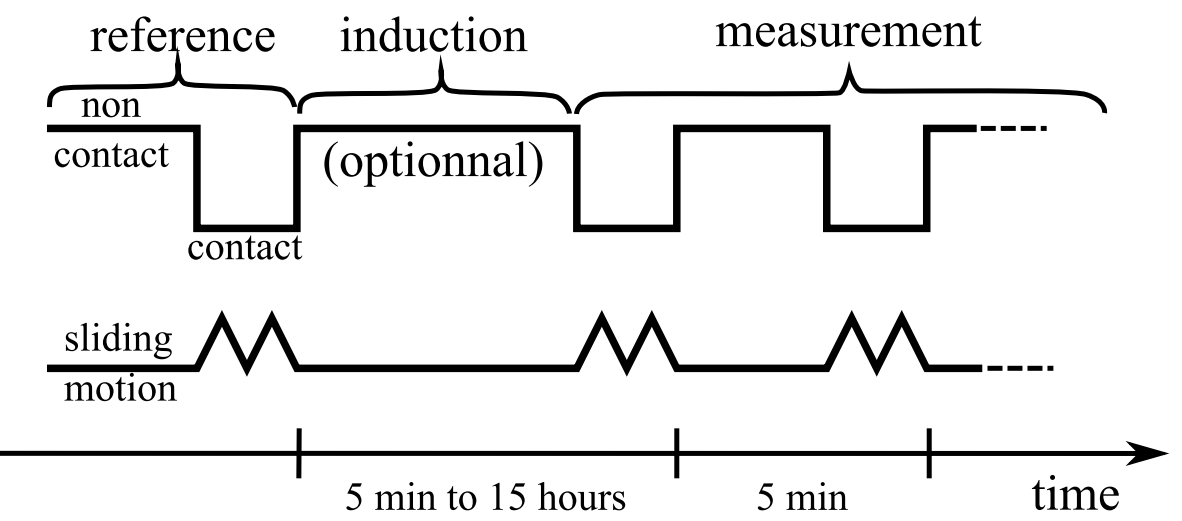

Fig. 2. Experimental protocol for the measurement of the kinetics of the onset of lubrication. The friction measurement proceeds by short friction runs separated by 5 min intervals during which the surfaces are kept far apart but submerged in the solution, in order to avoid drying problems. The measurement period is optionally preceded by an induction period during which the surfaces are kept far apart in the solution, without contact or friction measurement. The reference are individual runs performed initially in water and immediately after introducing the solution. 


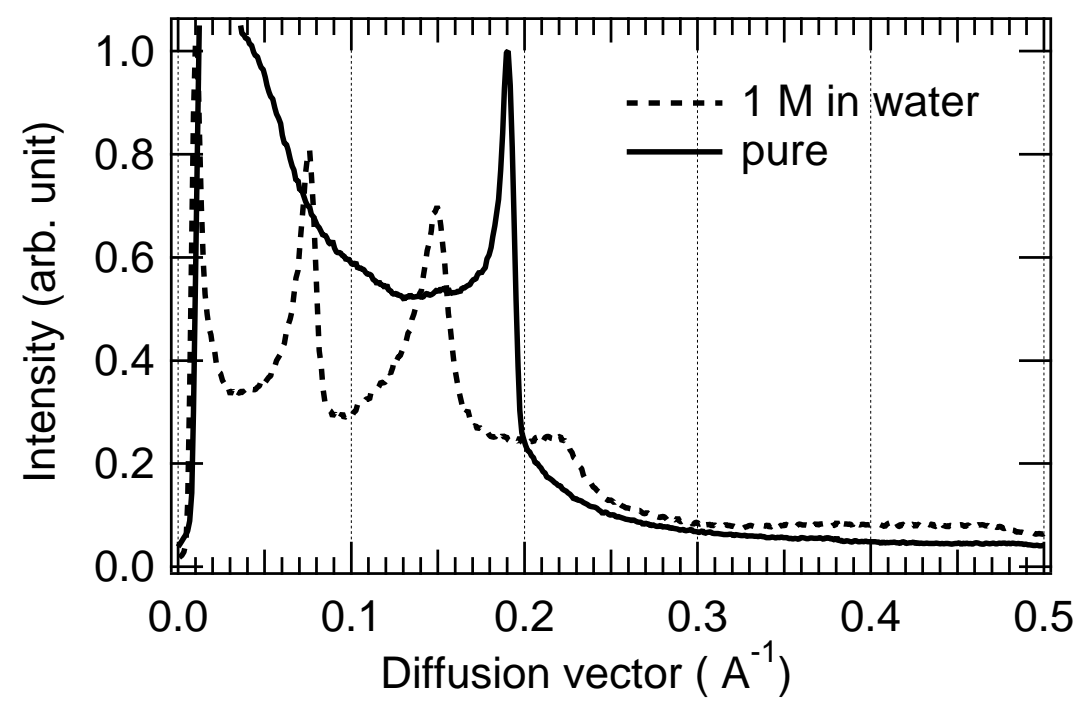

Fig. 3. Xray diffraction spectra for pure and diluted DOAIM. The fine diffraction peaks shifting to smaller wave vector with dilution demonstrate the presence of a lamellar phase.

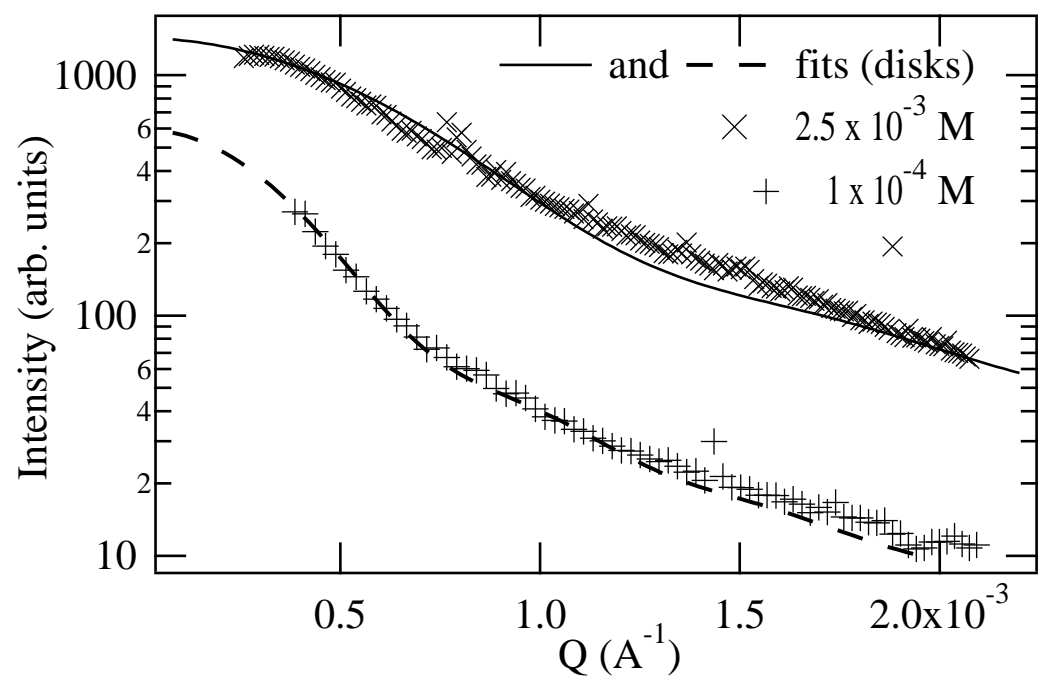

Fig. 4. Static light scattering of DOAIM at low concentrations with fits to diks shaped objects. The fits support the expected extremely diluted bilayer structure at these low concentrations. 


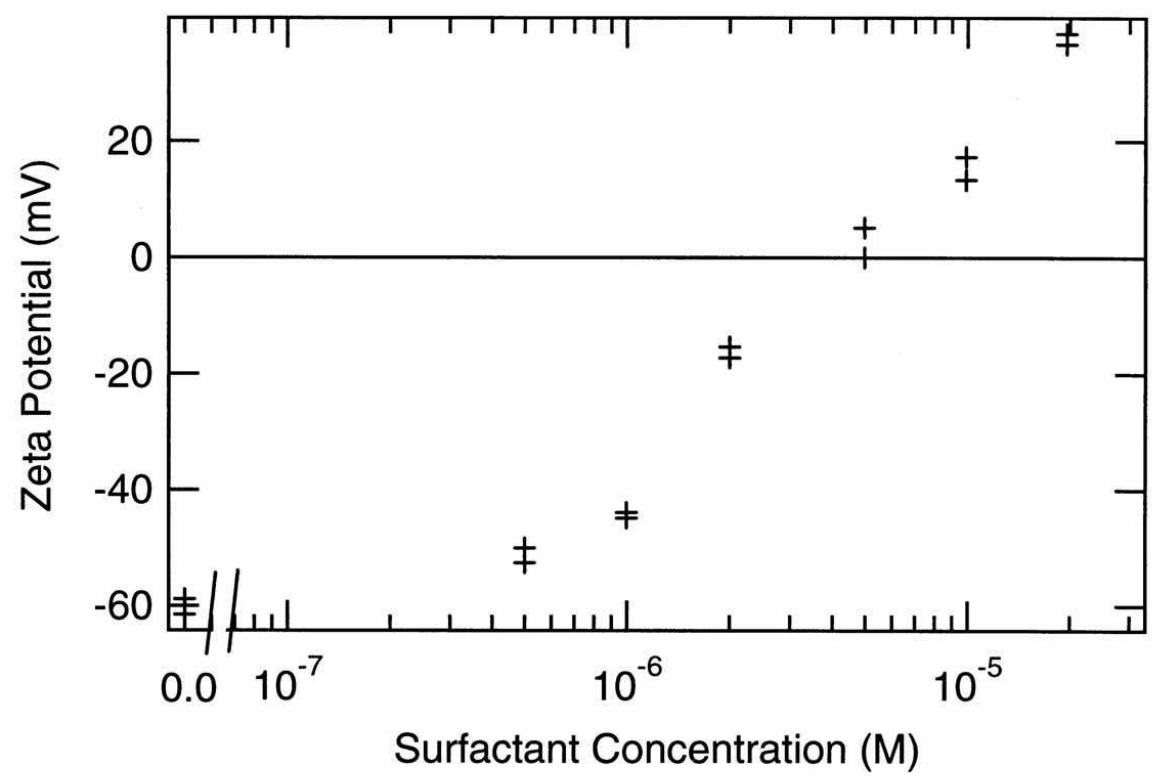

Fig. 5. Zeta potential as a function of surfactant concentration. The charge reversal is typical for the build-up of a surfactant bilayer at the surface. 


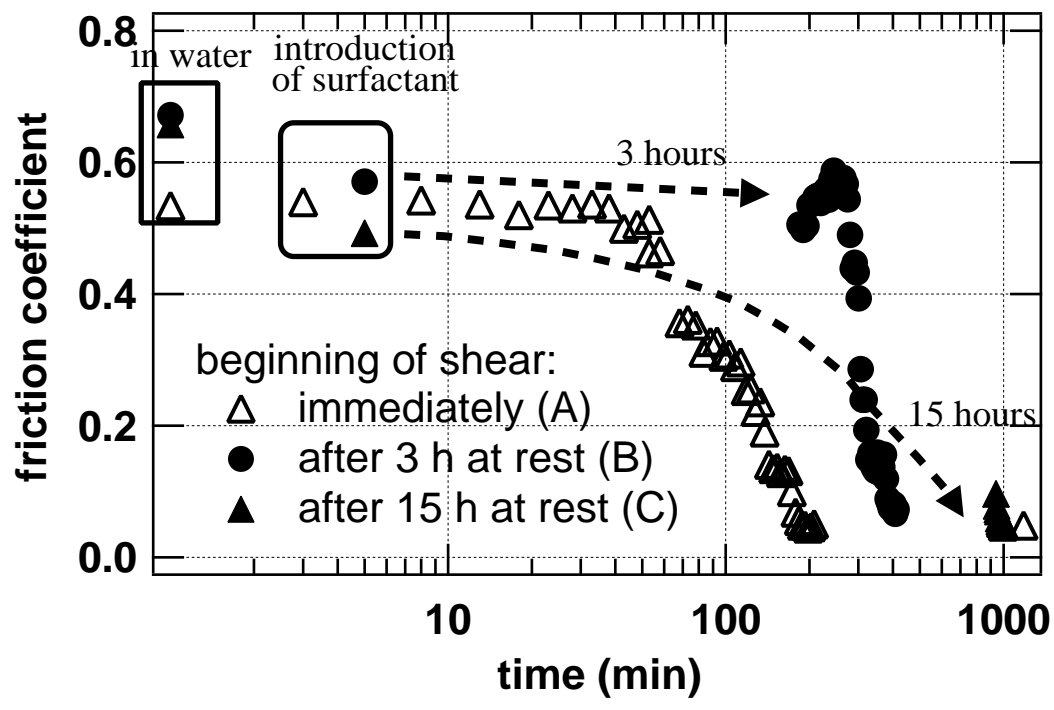

Fig. 6. Evolution of the friction coefficient vs immersion time $\left(\mathrm{C}=10^{-3} \mathrm{M}\right.$, $\left.\mathrm{v}=0.014 \mathrm{mms}^{-1}, \mathrm{P}_{m}=90 \mathrm{MPa}\right)$. In all cases, the friction coefficient is first measured in pure water, then immediately after introduction of the surfactant. In procedure $\mathrm{A}$, the friction coefficient is measured every 5 min. Procedures $\mathrm{B}$ and $\mathrm{C}$ are identical to procedure A, but the system is first left at rest for respectively 3 and 15 hours before the friction coefficient measurement starts. With procedures $\mathrm{A}$ and $\mathrm{B}$, the friction is initially high and stays constant for some induction period. Low friction is observed immediately after the end of the 15 hour wait period in procedure C. 


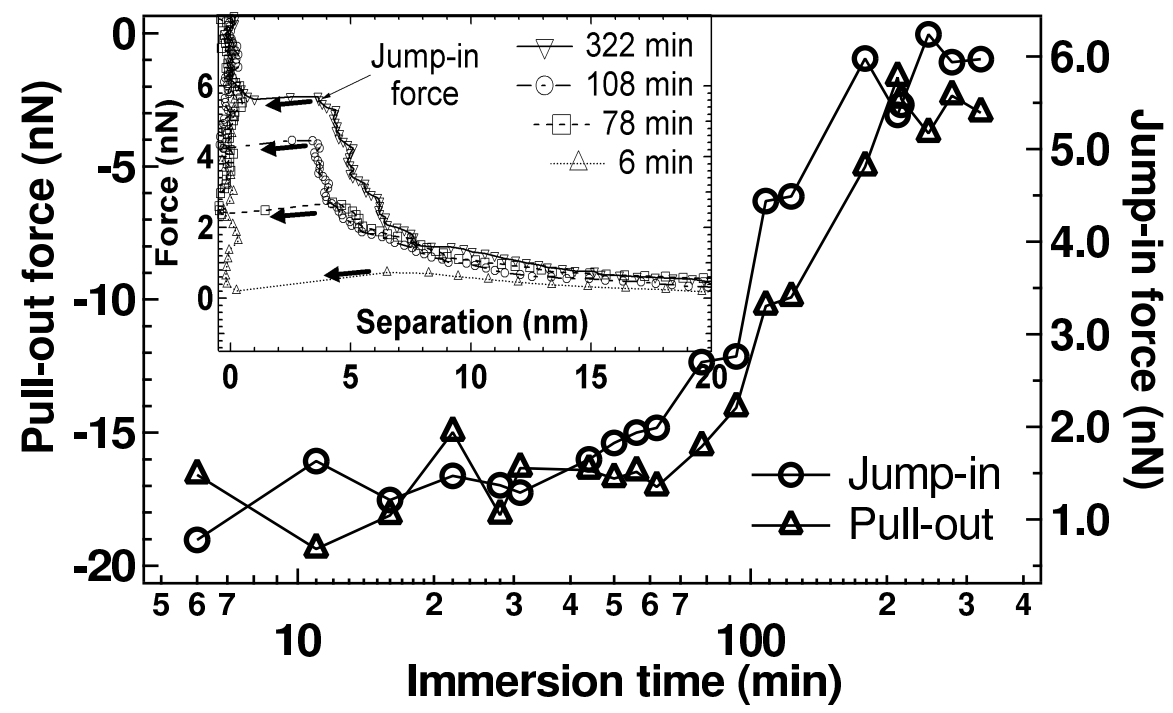

Fig. 7. Time evolution of the jump-in and of the pull-out forces between a silicon nitride tip and a silica surface $\left(\mathrm{C}=1 \times 10^{-3} \mathrm{M}\right)$. A few typical force vs distance curves are shown as inset.

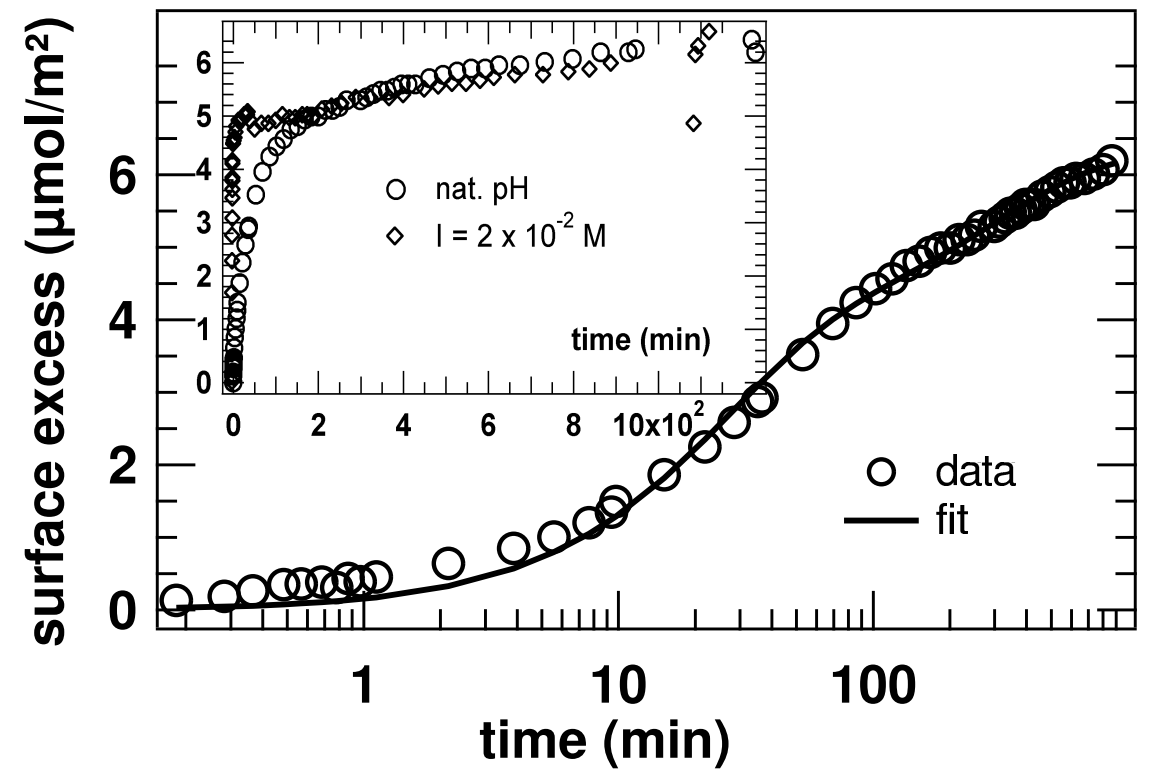

Fig. 8. Adsorption kinetics for DOAIM $\left(5 \times 10^{-4} \mathrm{M}\right)$ in pure water and fit to a double exponential function $\left(\tau_{1}=25 \mathrm{~min}, \tau_{2}=205 \mathrm{~min}\right)$. The inset compares the same data with the much faster kinetics at high ionic strength $\left(2 \times 10^{-2} \mathrm{M}\right)$ on a linear time scale. 


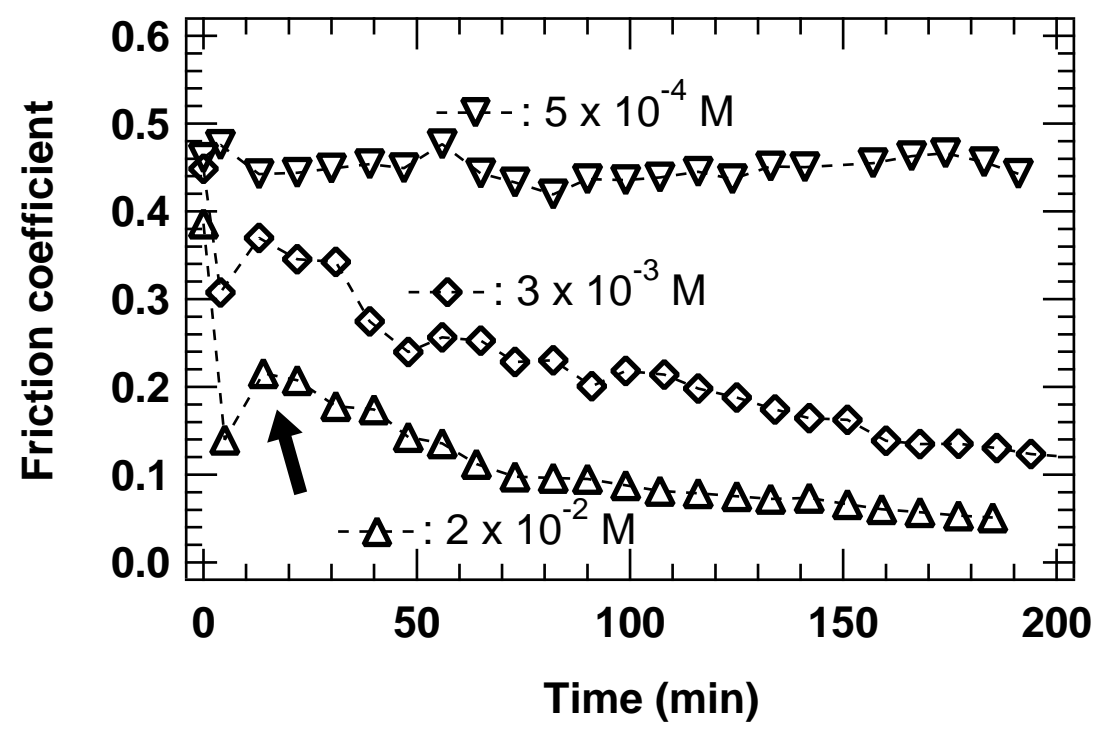

Fig. 9. Evolution of the friction coefficient versus immersion time $\left(C=5 \times 10^{-4} \mathrm{M}\right.$, natural $\mathrm{pH}$ ) at different ionic strengths. The arrow points to the friction spike observed after the initial lubrication effect of the surfactant.

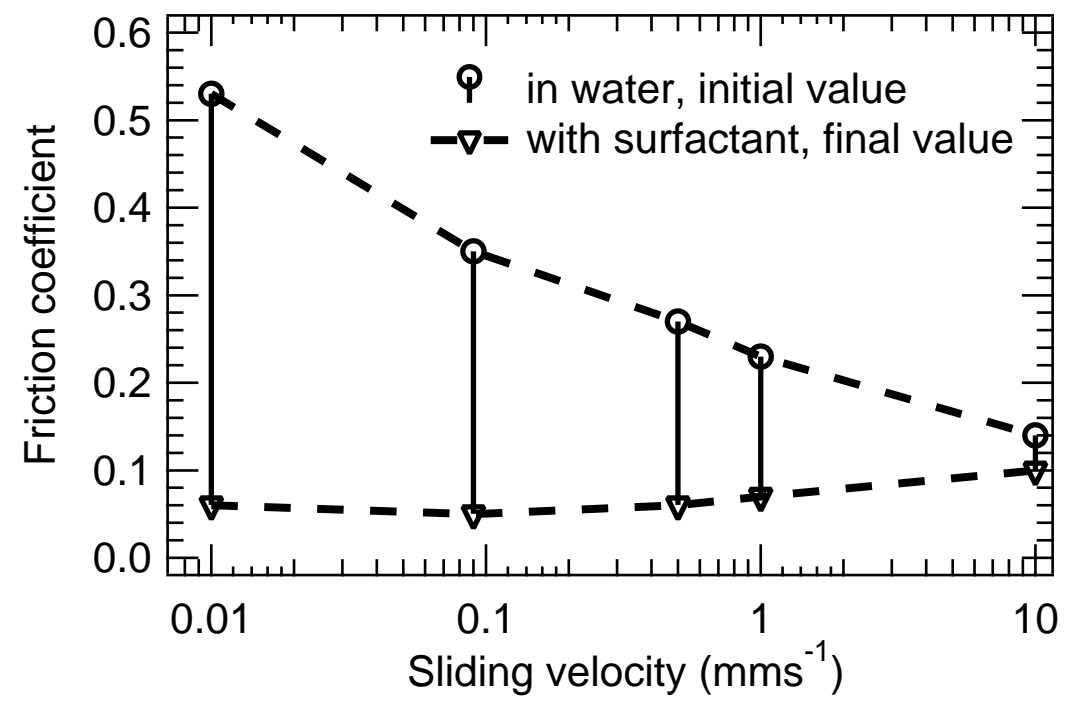

Fig. 10. Impact of shear velocity on the evolution of the friction coefficient between silica surfaces $\left(\mathrm{C}=10^{-3} \mathrm{M}, \mathrm{P}_{m}=320-340 \mathrm{MPa}, \mathrm{v}=10 \mathrm{mms}^{-1}\right)$. 


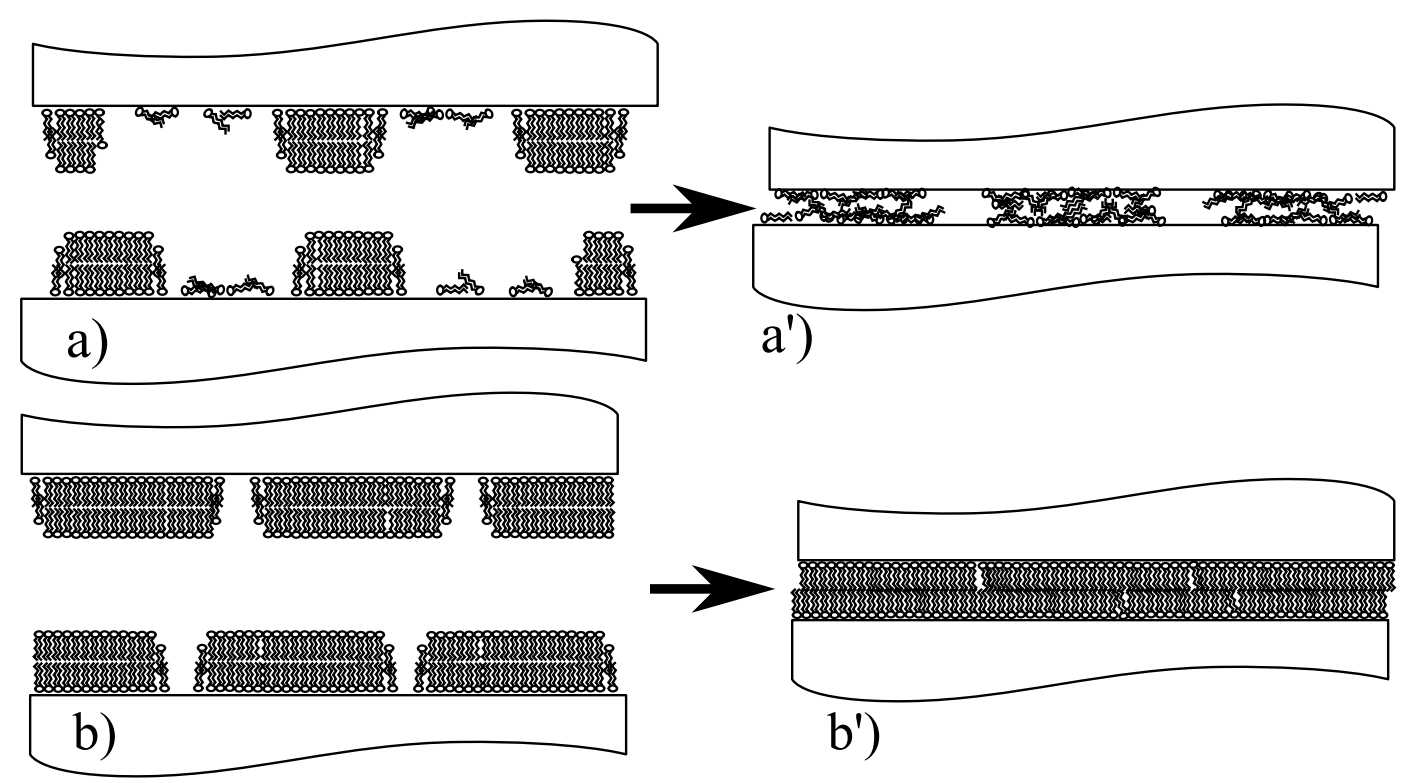

Fig. 11. Schematics of the surfactant instability for a highly defective (a, a') and an almost defect-free (b, b') bilayer. 